Forum $2021 \cdot 36: 276$

https://doi.org/10.1007/s12312-021-00960-x

(๑) Springer Medizin Verlag GmbH, ein Teil von Springer Nature 2021

\section{Prävention: den Fokus auf belegbaren Nutzen legen}

\author{
Thomas Seufferlein \\ Klinik für Innere Medizin I, Universitätsklinikum Ulm, Ulm, Deutschland
}

Kann man Krebs vorbeugen? Lässt sich das Krebsrisiko senken, wenn man gesund lebt, ausgewogen und vielseitig isst, Sport macht, nicht raucht und keinen Alkohol trinkt? Niemand mit einem medizinischen Hintergrund würde verneinen, dass eine gesunde Lebensweise statistisch gesehen das Risiko für schwere chronische Erkrankungen, etwa koronare Herzerkrankungen, Krebs, Diabetes oder Bluthochdruck, senken kann. Was genau mit einer gesunden Ernährung gemeint ist, wer ein besonderes Risiko für eine dieser Erkrankungen aufweist und welche Stellschraube im Einzelfall zur Senkung des Krankheitsrisikos gedreht werden muss, lässt sich anhand der vorhandenen Evidenz jedoch meist nicht so leicht beantworten.

Vielleicht ist das der Grund dafür, dass der Appell, gesund zu leben, in der Bevölkerung nur schwer Gehör findet. Studien, die die Evidenz für die Wirksamkeit ei-
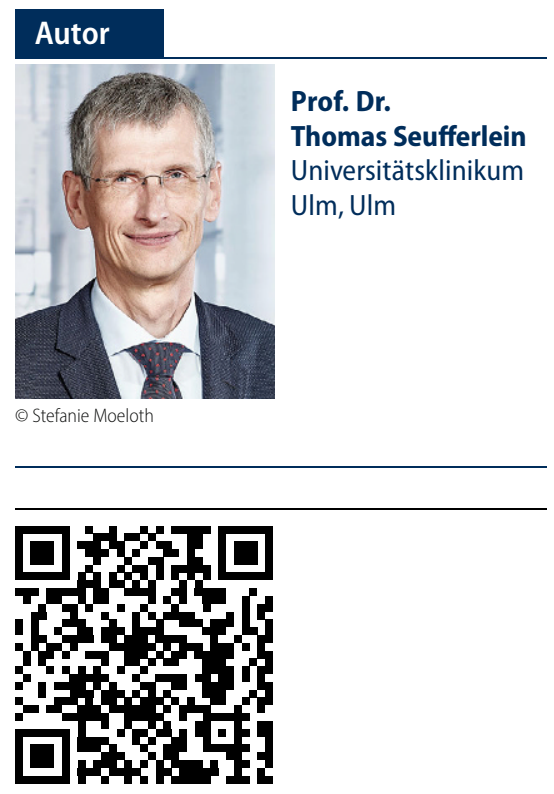

QR-Code scannen \& Beitrag online lesen ner bestimmten Präventionsmaßnahme liefern, dauern meist sehr lange. Es ist schwierig, Outcome-Parameter über eine lange Zeit konsequent zu verfolgen. Darüber hinaus geht es häufig um eine Veränderung des Lebensstils, um das Loslassen lieb gewordener Gewohnheiten. Selbst wenn medizinische Evidenz für die Wirksamkeit einer Vorsorgemaßnahme vorhanden ist, kennen wir noch lange nicht die psychologischen und sozialen Barrieren, die für ihre mangelnde Akzeptanz in der Bevölkerung sorgen. Kritische Stimmen bemängeln ohnehin, dass unsere Gesundheitskommunikation meist Zielgruppen mit einer hohen Gesundheitskompetenz anspricht. Doch was ist mit den anderen - Menschen in prekären Verhältnissen, Kinder und Jugendliche, die Gesundheitskompetenz erst noch aufbauen müssen, Menschen mit einer anderen Muttersprache oder einem anderen kulturellen Hintergrund?

Um die Krebsprävention voranzubringen, brauchen wir sicher mehr gezielte medizinische Forschung. Genauso wichtig sind soziologische und sozialpsychologische Ansätze, die Aufschluss darüber geben, wie die verschiedenen Zielgruppen in der Bevölkerung am besten erreicht werden können. Das Deutsche Krebsforschungszentrum (DKFZ) und die Deutsche Krebshilfe planen gemeinsam die Errichtung eines Nationalen Krebspräventionszentrums. Das ist ein guter Anfang.

Die Deutsche Krebsgesellschaft (DKG) als Fachgesellschaft kann viel dazu beitragen, diese Anstrengungen zu unterstützen: durch eine Haltung, die den Fokus auf Interventionen mit belegbarem Nutzen legt und durch die konsequente Berücksichtigung der Prävention in den Leitlinien des Leitlinienprogramms Onkologie. Hilfreich ist auch unsere multidisziplinäre
Kompetenz in der Betreuung der Betroffenen an der Schnittstelle zwischen Früherkennung und Therapie. Beispiele sind die Dysplasiezentren und -sprechstunden in unseren zertifizierten gynäkologischen Zentren sowie die FBREKZentren, die Frauen mit einem genetisch bedingten Risiko für Brust- und Eierstockkrebs beraten und betreuen, dabei eng mit den DKG-zertifizierten Brustkrebszentren zusammenarbeiten und hervorragende Forschungsarbeit leisten. Die Beratungsstellen der Landeskrebsgesellschaften sind außerdem gute Multiplikatoren für Gesundheitsinformationen - eine wichtige Basis für Präventionskampagnen. An der Ausrichtung der Nationalen Krebspräventionswoche im September 2021 beteiligt sich neben dem DKFZ und der Deutschen Krebshilfe erstmals auch die Deutsche Krebsgesellschaft. Denn schließlich können wir sehr viel mehr erreichen, wenn wir unsere Kräfte bündeln.

Ihr Thomas Seufferlein

Präsident der Deutschen Krebsgesellschaft

Korrespondenzadresse

Prof. Dr. Thomas Seufferlein

Klinik für Innere Medizin I, Universitätsklinikum Ulm

Albert-Einstein-Allee 23, 89081 Ulm,

Deutschland

thomas.seufferlein@uniklinik-ulm.de

Interessenkonflikt. T. Seufferlein gibt an, dass kein Interessenkonflikt besteht. 